demands that rescue operations shall continue until every man is accounted for, dead or alive.

We might expect a book on such an enthralling subject to be itself enthralling. Alas, this is not so. Professor Lucas has succeeded in producing a work which, though packed with fascinating material, is nearly unreadable. The writing is so prolix and loaded with jargon that every page is a toil to read. It would be an interesting exercise to translate some passages into decent English prose: for example, the limpid conclusion: "Anticipatory socialization involving non-structured, non-repetitive behaviour in an unknown environment introduces anxiety and role performance insecurity". This we might endure, even willingly, if our understanding were enhanced. But we may reasonably ask: do the priestly polysyllables of sociological writing hold any insights into the behaviour of men under great stress that we could not have obtained, more briefly and vividly, by asking any intelligent sergeant of Marines? The answer seems to be "no".

M. Hammerton

\section{SECRETORY PHYSIOLOGY}

The Exocrine Glands

Edited by S. Y. Botelho, F. P. Brooks and W. B. Shelley. (Satellite Symposium of the 24th International Congress of Physiological Sciences.) Pp. $\mathrm{xx}+280$. (University of Pennsylvania Press: Philadelphia; Oxford University Press: London, April 1970.) 190s.

THIs volume contains a selection of research papers on the secretory mechanism of exocrine glands. Much of the work reported is exciting and of high quality scientifically, but there may well be those who would find it hard to justify publication of yet another expensive verbatim report of the proceedings of an international symposium. Tho references appended to each paper indicate that many of the findings have been recently published, or are in the process of being published, in the scientifie literature. What therefore does one look for in such a book? The general reader will find it of little use as a source of information on exocrine glands; there is no subject index to help him. Of more value than most of the other papers in this respect, however, is the review article in the first section on the reptilian salt glands. For the specialist, the perspective of modern research on glandular physiology is momentarily displayed, though in this rapidly advancing field of research much will have crystallized or have been superseded in a few years' time. One sixth of the volume records the discussions which have the quality of caution but a propensity for introducing further papers in miniature.

The organizers of the symposium and the editors of this volume have broadly subdivided the contributions into three different aspects of the secretory process: the secretion of electrolytes and water, the secretion of organic substances, and the neural, hormonal and pharmacological control. If one objective of the symposium was to explore common mechanisms in exocrine secretory procosses, little factual headway can be discerned. Nonuniformity seems to be the rule even, for example, in the composition of primary secretions. In the submaxillary gland it is a plasma ultrafiltrate, in the atrichial sweat gland the precursor fluid is hypertonic and in the pancreatic acini the primary fluid is isosmolal with plasma but rich in bicarbonate. One contributor deduced from micropuncture studies that the primary pancreatic fluid is, on the contrary, low in bicarbonate; the absence of discussion of this anomaly perhaps speaks volumes for our ignorance about the most fundamental ionic events in secretory cells. The sophistication of microtechniques in modern research is clear to see; those methods developed in earlier work on the salivary gland are now being applied with success to the pancreas and sweat glands. The section on secretion of organic substances includes a variety of themes on the very frontiers of enquiry. It includes consideration of the solvent-drag effect in nonelectrolyte transport, the hormonal control of protein synthesis and transport in pancreatic acinar cells and an investigation of the synthesis of $\gamma \mathrm{A}$ immunoglobulin in human salivary glands. In the final section on neurohumoral control, the difficulties with inter-species differences especially in sweat glands, and of deciding the cause of an observed effect of autonomic nerve stimulation, are made abundantly plain.

Professor A. S. V. Burgen has provided a short introduction and general summary which are both succinct and provocative.

K. J. Collins

\section{MILK FLOW}

\section{Lactogenesis}

The Initiation of Milk Secretion at Parturition. Edited by M. Reynolds and S. J. Folley. (Proceedings of a Symposium held at the University of Pennsylvania, August 24, 1968.) Pp. xii +269 . (University of Pennsylvania Press: Philadelphia, March 1970.) \$12.50.

LACTOGENESIS is a term which should be abandoned or redefined. It encourages the assumption that "the initiation of a copious flow of milk at or about the time of parturition" occurs in one step. In fact, at least two steps can be distinguished. One is the transition of the mammary parenchymal cells from a non-secreting to a secreting state. This change is well defined in the electron microscope and is accompanied by increases in the activities of many enzymes. In the rat and mouse it occurs only a few hours before parturition, but in the cow apparently over a week before. That this transition is induced by hormones is vividly illustrated when prolactin is injected into a teat duct of a pregnant rabbit: the associated gland becomes engorged with milk, although its volume is a small fraction of the daily yield of a suckled gland.

Another step in "lactogenesis" is the stimulation of an appreciable yield of milk from cells which have undergone the previous transition. This is clearly initiated by the withdrawal of milk, and caused by a mechanism which is independent of changes in hormone and nutrient concentrations of blood. This is shown by the experiments of Dr Dodd and his colleagues at Shinfield in which milking of two quarters of a lactating cow can be stopped and successfully restarted some weeks later, without the yield from the quarters which are milked throughout being affected. These experiments are not mentioned in this volume, possibly because of a false belief that they necess. arily conflict with the classical demonstration of Selye that all glands of a lactating rat are maintained in a secreting state when milk is removed from only half of its teats by suckling.

At symposia, free thought about these distinctions seems to be stifled by an unspoken requirement to consider lactogenesis as a one-step change induced by hormones. Hence the papers in this volume largely relate to the transition which is induced by hormones; namely, the conversion of non-secreting to secreting cells, and there is little concern with how "copious" is the resulting flow of milk. Apart from this limitation the papers give an excellent summary of our knowledge of this transition by twenty-eight leading workers. Changes in ultrastructure, composition and enzyme content of the parenchymal cells with the onset of sccretion are discussed in detail. Two interesting papers discuss changes in blood flow through the mammary gland and other organs at parturition, and two deal with milk ejection. Finally, hormone changes involved in the initiation of secretion are thoroughly considered both from evidence in living animals and from organ cultures. 